\title{
High Expression Levels of KIF18A is an Independent Predictor of Poor Survival in Glioma: A Study Using Multi Public Data
}

\author{
Haigang Chang \\ Department of Neurosurgery, General Hospital of Ningxia Medical University \\ Yaxiao Wang \\ Xinxiang Medical University First Affiliated Hospital \\ Yan Li \\ Xinxiang Medical University First Affiliated Hospital \\ Weilong Yang \\ Ningxia Medical University \\ Lei Wang \\ Ningxia Medical University \\ Pengju Ma \\ Xinxiang Medical University First Affiliated Hospital \\ Xinxiao Li \\ Ningxia Medical University \\ Wenke Zhou \\ Xinxiang Medical University First Affiliated Hospital \\ Zhendong Liu \\ Henan Provincial People's Hosptial \\ Feng Wang ( nxwwang@163.com) \\ General Hospital of Ningxia Medical University
}

Research article

Keywords: KIF18A, Glioma, Gene expression, Prognosis

Posted Date: September 2nd, 2020

DOI: https://doi.org/10.21203/rs.3.rs-64417/v1

License: (a) (i) This work is licensed under a Creative Commons Attribution 4.0 International License. Read Full License 


\section{Abstract}

Background: Kinesin family member 18A (KIF18A) belongs to a member of the kinesin superfamily and is significantly overexpressed and abnormally functions in a variety of cancers. However, the expression profiling and the role of KIF18A has so far remained unclear in glioma. To investigate the relationship of KIF18A with clinical practice and its role in gliomas, the data from multi public datasets were used and analyzed in present study.

Methods: Patients with gliomas were enrolled from CGGA RNA-seq, TCGA-seq, CGGA-microarray, GSE4290 and GSE50161 databases. The expression of KIF18A in glioma and normal tissues was analyzed through Wilcoxon rank sum test, and the relationship between KIF18A and clinicopathological features was evaluated using logistic regression. The effect of KIF18A expression in the survival of glioma patients was explored by Kaplan-Meier and Cox analyses. Gene set enrichment analysis was also conducted to annotate biological function of KIF18A.

Results: KIF18A expression was significantly increased in glioma tissues compared with the normal counterparts. High KIF18A expression shortened the overall survival in all grades gliomas and the survival period of patients with WHO grade $₫$ glioma, but

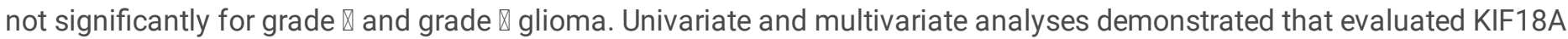
expression is closely related to poor prognosis. Increased KIF18A expression was significantly correlated grades, age, IDH wide type and may serve as a biomarker of poor prognosis in gliomas. GSEA showed that the KEGG_DNA_REPLICATION, the KEGG_CELL_CYCLE, the KEGG_MISMATCH_REPAIR and the KEGG_NUCLEOTIDE_EXCISION_REPAIR were differentially enriched in KIF18A high expression phenotype.

Conclusions: High expression KIF18A might be potential biomarker for the diagnosis and an independent risk factor for poor prognosis in glioma.

\section{Background}

Glioma is the main primary malignant tumor of the central nervous system [1], which has high mortality and high recurrence rate, heavy economic burden to individuals, families and society [2]. At present, the main treatment includes maximum tumor resection and adjuvant chemotherapy and radiotherapy, but the long-term prognosis is still poor [3]. It is urgent to explore the mechanism of the occurrence and development of glioma from the level of gene and molecular signaling pathway, which may become a new direction of glioma treatment and bring new hope for glioma patients. Over the past decade, neuroscientists and oncologists have identified many genes, factors, such as EGFR and p53 molecules and associated signaling pathways that affect the development and prognosis of gliomas [4,5]. Furthermore, specific targeting antibodies have been developed for specific molecules or factors to treat gliomas, such as anti EGFR antibodies, bevacizumab. Although it has been used in clinical practice, there are not enough studies to prove that it can improve the overall survival rate of patients. At present, the understanding of the occurrence, development and treatment mechanism of glioma is still insufficient. It is necessary to continue to explore reliable biomarkers to predict the early occurrence of gliomas and as new therapeutic targets, and to develop safer and earlier diagnosis and prognosis prediction measures to improve the overall treatment effect of glioma patients.

Kinesin is a microtubule-based motor protein that was first found in the mammalian brain [6] and functions at different stages of cell division, intracellular vesicles and organelle transport, and microtubule movement [7, 8]. An increasing number of kinesin family members are thought to be involved in the development and progression of tumors and are closely related to the invasion, metastasis and poor prognosis of multiple cancers [9-12].

KIF18A belongs to the kinesin-8 family and is a multifunctional protein involved in a variety of cellular functions, including cell division, motility, microtubule dynamics, organelle transport, and neural signal transduction. Notably, some recent reports suggest that KIF18A are also involved in the process of multiple tumors. Kif18A is associated with the occurrence and development of liver cancer [13], breast cancer [14], ovarian cancer [15] and can be used as potential biomarkers for breast cancer [16], colorectal [17] and hepatocellular carcinoma [18]. In contrast, the expression of kif18a is low in gastric cancer, 
which is associated with poor prognosis [19]. Therefore, Kif18A expression patterns appear to differ between different cancer types, and specialized studies on KIF18A expression patterns in gliomas and their clinicopathological significance are rare. Only one study, using microarrays, found that KIF18A18a was consistently expressed in glioblastoma stem cells cultures and the gene expression of KIF18A was confirmed in clinical samples [20]. We speculate that KIF18A is involved in the occurrence and development of gliomas. However, the relationship between KIF18A and gliomas is still not entirely clear, and further research is needed.

Therefore, this study systematically explored the expression of KIF18A in glioma and its effect on patient prognosis through multiple datasets using multiple biological analysis methods.

\section{Methods}

\section{Data collection and bioinformatic analysis}

Microarray information of 268 glioma cases were download from CGGA dataset (Additional file 1: Table S1). Clinical and RNASeq data was obtained from the CGGA database, which includes 218 WHO grade II, 240 WHO grade III, and 291 WHO grade IV (Additional file 2: Table S2).

mRNAseq data of 653 glioma patients were collected in TCGA database. Clinical data were also obtained from TCGA database, which includes 377 male and 276 females, ranging from WHO grade II to WHO grade IV (Additional file 3: Table S3).

Two raw gene expression datasets [GSE4290 (77 tumor and 23 normal tissue), GSE50161 (34 tumora and 13 normal)] downloaded from the Gene Expression Omnibus (GEO) database were used to validate the association between KIF18A expression and glioma outcome and the limma package in the $\mathrm{R}$ language (version 3.6.1) was performed. A fold change (FC) value of $(\log F C)>1$ and a $P$ value $<0.05$ are screening criteria.

GEPIA provides key interactive analysis and customization functions, including tumor/normal differential expression profile analysis, profiling, pathological staging, patient survival analysis, similar gene detection analysis and dimensionality reduction analysis. The gene expression profile of KIF18A across all tumor samples and paired normal tissues was download from public database GEPIA (http://gepia.cancer-pku.cn/index.html).

The expression of KIF18A in normal cerebral cortex, low- and high- grade glioma, identified by immunohistochemistry staining, was from the Human Protein Atlas (https://www.proteinatlas.org/).

\section{Gene set enrichment analysis}

In order to explore the potential molecular mechanism of KIF18A expression influencing the prognosis of glioma, GSEA was used to detect whether there was a statistically significant difference between the high and low KIF18A expression groups. Gene sets with a normal $p$-value $<0.05$ and false discovery rate $(F D R)<0.25$ were considered to be significantly pathways enriched.

\section{Statistical analysis}

R (v.3.6.1 version) was used for statistical analysis. Wilcox test was used to identify KIF18A expression in glioma and nontumor brain tissues. The relationship between KIF18A expression and patient OS was analyzed by Cox regression and KaplanMeier method, and survival curves was drawn. The relationship between clinical information and the expression of KIF18A in gliomas was analyzed by Wilcox or Kruskal test.

\section{Results}

\section{Expression of KIF18A in tumors and normal tissues}


The expression of specific genes in the normal tissues of specific tumors compared with tumor tissues can be analyzed through GEPIA. GEPIA analysis results showed that the expression of KIF18A was significantly increased in many tumor tissues, such as BLCA, BRCA, CESC and so on. Meanwhile, the results demonstrated that the expression of KIF18A increased in GBM (Figure 1a).

In order to further explore the expression of KIF18A in glioma, two GEO datasets (GSE4290 and GSE50161) were enrolled and analyzed. The results demonstrated that the expression of KIF18A in tumor sample tissues up-regulated significantly, compared to normal brain tissue (Figure $1 \mathrm{~b}$ and $\mathrm{c}$ ).

Mutiple different sources of data contain 1781 glioma and 36 normal tissues. We found that the expression of KIF18A increased in glioma, but its role in glioma needs further study.

\section{Increased KIF18A expression reduces overall survival of glioma patients}

In order to explore the function of KIF18A expression in glioma, we first studied whether KIF18A expression affects the survival of patients with glioma. Kaplan-Meier method was used in this study. A total of 1670 glioma patients had KIF18A expression information from CGGA RNA-seq $(n=749)$, CGGA microarray $(n=268)$ and TCGA RNA-seq $(n=653)$ sets. After analyzing each data set, we found that the overall survival time of patients with higher KIF18A expression was shortened in all grades gliomas

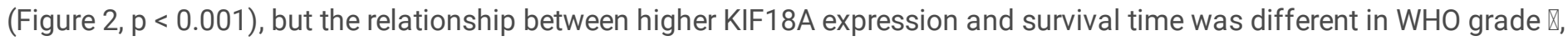
WHO grade $\nabla$ and $W H O$ grade $\otimes$ gliomas. In CGGA RNA-seq data set, for WHO grade $\otimes$ (Figure $2 b, p=0.014$ ) and WHO grade $\nabla$ (Figure $2 \mathrm{c}, \mathrm{p}<0.001$ ) glioma patients, higher KIF18A expression shortened the survival period, but for WHO grade $\otimes$ (Figure $2 \mathrm{~d}$, $p=0.101$ ) glioma patients, there was no significant difference between high and low expression of KIF18A patients. In CGGA

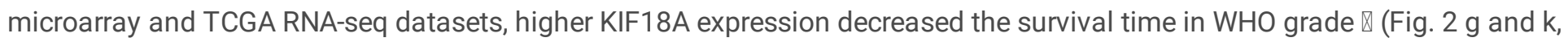
$p<0.01$ ) glioma patients, but had no significant effect on WHO grade $\otimes$ (Figure $2 f, p=0.601$ and $j, p=0.66$ ) and WHO grade $\otimes$ (Figure $2 \mathrm{~h}, \mathrm{p}=0.38$ and $\mathrm{l}, \mathrm{p}=0.402$ ) glioma patients. From these data, we found that higher KIF18A expression shortened the overall survival in glioma and the survival period of patients with WHO grade $\nabla$ glioma, but the effect on the survival period of patients with $\mathrm{WHO}$ grade $\otimes$ and $\mathrm{WHO}$ grade $\otimes$ glioma was not statistically significant.

\section{KIF18A is an independent prognostic factor and increased KIF18A expression is associated with poor prognosis}

The high expression of KIF18A can reduce the overall survival of glioma patients. Whether KIF18A is an independent prognostic factor needs to be further verified. In this study, univariate and multivariate regression analysis were used to analyze the three data sets. In CGGA RNA-seq data set, univariate analysis showed that KIF18A (HR $=2.201 ; 95 \% \mathrm{Cl}=1.954-2.479 ; \mathrm{P}$ $<0.001$ ), prs_type, histology, grade, age, chemo, idh_mutation and 1p19q_codelization could predict the overall survival in all grades gliomas (Figure 3a). In multivariate regression analysis, KIF18A (HR $=1.372 ; 95 \% \mathrm{Cl}=1.186-1.586 ; \mathrm{P}<0.001)$ still significantly affected the prognosis with adjusting prs_type, histology, grade, age, chemo, idh_mutation, 1p19q_code selection (Figure 3B). Univariate analysis showed that KIF18A (HR = 1.649; 95\% Cl = 1.486 - 1.830; P < 0.001), TCGA_subtypes, PRS_type, histology, grade, age and IDH_mutation could predict the overall survival in all grade gliomas in the CGGA microarray

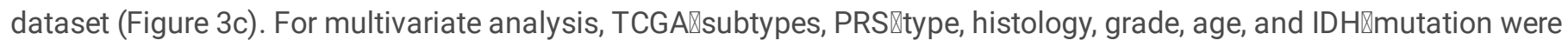
corrected, and KIF18A ( $\mathrm{HR}=1.372 ; 95 \% \mathrm{Cl}=1.203-1.566 ; \mathrm{P}<0.001)$ was significant (Figure 3d). Univariate analysis in TCGA RNA-seq showed that KIF18A ( $H R=1.432 ; 95 \% \mathrm{Cl}=1.330-1.542 ; \mathrm{P}<0.001)$, age and grade were significantly associated with overall survival (Figure 3e). In multivariate regression analysis, with age and grade adjusting, $\mathrm{KIF} 18 \mathrm{~A}(\mathrm{HR}=1.224 ; 95 \% \mathrm{Cl}=$ 1.067 - 1.404; $\mathrm{P}<0.001$ ) still had significant effect on prognosis (Figure 3f). Above data show that KIF18A plays an important role in glioma progression and is closely related to poor prognosis.

\section{KIF18A is a biomarker of glioma}

To assess KIF18A diagnostic value in glioma, the receiver operating characteristic curve were obtained from three datasets. In CGGA RNA-seq data set, the areas under the curves (AUC) for one year, three year and five year are $0.677,0.764$ and 0.791 respectively. AUC were $0.739,0.819$ and 0.780 respectively in CGGA microarray data set and $0.801,0.860$ and 0.818 respectively in TCGA RNA-seq set. The diagnostic value of KIF18A in different grades gliomas was further analyzed. The 
results are shown in Figure 4 (b, c, d, f, g, h, j, k and I). These results show that KIF18A has reliable predictive and diagnostic value in glioma.

\section{Relationship between KIF18A expression and clinical characteristics of glioma}

The relationship between the KIF18A expression and the clinical characteristics of glioma was analyzed from three data sets. In CGGA RNA-seq data set, the increased expression of KIF18A is closely related to WHO grade, chemo status, age, PRS type, IDH mutation status, 1p19q coding status and histology, as shown in Figure 5 (a, b, c, d, e, f and g). In CGGA microarray data set, the increased KIF18A was significantly related to WHO grade, age, IDH mutation status and history. TCGA RNA-seq data set analysis showed that increased KIF18A expression was significant in high-grade gliomas and in patients aged over 51 . These results suggested that increased KIF18A expression is more likely to occur in high-grade, elderly, IDH wide type glioma patients.

\section{Expression of KIF18A protein in glioma}

To further explore the expression of KIF18A protein in glioma, immunohistochemical sections of normal brain tissue (Figure $6 a)$, low (Figure 6b), and high grade gliomas (Figure 6c) were downloaded from the human protein atlas. The data showed that the expression of KIF18A in gliomas was enhanced compared to normal brain tissues, and the expression of KIF18A was the most significant in high-grade gliomas.

KIF18A related signaling pathways based on GSEA

Gene set enrichment analysis was used to identify glioma related signaling pathways between low and high KIF18A expression in three data sets. GSEA showed significant differences (FDR < 0.25, NOM p-val < 0.05) in enrichment of MSigDB Collection (c2.cp.biocarta and h.all. v6.1. symbols). We selected four pathways, including the KEGG_DNA_REPLICATION, the KEGG_CELL_CYCLE, the KEGG_MISMATCH_REPAIR and the KEGG_NUCLEOTIDE_EXCISION_REPAIR, showed significantly differential enrichment in KIF18A high expression phenotype based on NES, NOM P value, and FDR value (Figures 6a - d; Table 1), which suggested KIF18A play a special role in the development of glioma.

Table 1.The gene set enriches the high KIF18A expression phenotype.

\begin{tabular}{|llllllllll|}
\hline & \multicolumn{2}{c}{ CGGA RNA-seq } & \multicolumn{3}{c}{ CGGA microarray } & \multicolumn{3}{c|}{ TCGA RNA-seq } \\
\hline Gene set name & NES & $\begin{array}{l}\text { NOM } \\
\text {-val }\end{array}$ & $\begin{array}{l}\text { FDR } \\
\text { q-val }\end{array}$ & NES & $\begin{array}{l}\text { NOM } \\
\text { p-val }\end{array}$ & $\begin{array}{l}\text { FDR q- } \\
\text { val }\end{array}$ & $\begin{array}{c}\text { NES } \\
\text { NOM }\end{array}$ & $\begin{array}{l}\text { FDR } \\
\text { p-val } \\
\text { q-val }\end{array}$ \\
\hline KEGG_DNA_REPLICATION & 1.856 & 0 & 0.154 & 1.880 & 0.006 & 0.0423 & 1.912 & 0 & 0.017 \\
\hline KEGG_CELL_CYCLE & 1.924 & 0.001 & 0.116 & 2.123 & 0 & 0.002 & 2.134 & 0 & 0.005 \\
\hline KEGG_MISMATCH_REPAIR & 1.8019 & 0 & 0.119 & 1.873 & 0.002 & 0.0371 & 1.931 & 0 & 0.016 \\
\hline KEGG_NUCLEOTIDE_EXCISION_REPAIR & 1.774 & 0.001 & 0.114 & 1.764 & 0.009 & 0.093 & 1.954 & 0 & 0.013 \\
\hline
\end{tabular}

NES: normalized enrichment score; NOM: nominal; FDR: false discovery rate. Gene sets with NOM P-value $<0.05$ and FDR qvalue $<0.25$ were considered as significantly enriched.

\section{Discussion}

Despite great progress in the treatment for glioma, including surgery, radiotherapy and chemotherapy, and even comprehensive and individualized treatment, the overall prognosis of glioma patients remains poor. At present, there is still a lack of in-depth understanding of the exact causes and processes of gliomas. How to improve the early diagnosis of gliomas and how to improve the comprehensive treatment effect in addition to surgery is still a major problem in the world. Global scientists hope that targeted therapy for the major molecular markers of glioma has not effectively improved the survival rate of patients. 
Therefore, it is urgent and important to continue to search for some new targeted genes that can help the early diagnosis and evaluate the prognosis of glioma patients and improve the cure rate.

Abnormal and uncontrolled proliferation associated with tumor growth requires activation of a variety of key proteins. In recent years, research on tumor therapy has focused on proteins involved in mitotic regulatory events [21-23]. Mitotic inhibitors, including taxanes and vincristine, act on microtubules and achieve varying degrees of success in treating various types of cancer [24]. Specific kinesin motor proteins were considered as key proteins and a potential therapeutic target in the regulation of mitotic events $[10,25]$. Kinesins play an important role in mitosis, and its abnormal expression or dysfunction can lead to malignant transformation of cells [14, 26-28]. Due to their important role in mitosis, kinesin family has become the target of chemotherapy intervention in malignant tumor [29].

KIF18A is a member of the kinesin superfamily of microtubule-associated molecular motors that use hydrolysis of ATP to produce force and movement along microtubules [30,31]. KIF18A plays an important role in chromosome arrangement during mitosis, and chromosome instability is the basis of tumor transformation and metastasis progression [32-34].

Although KIF18A expression has been detected in a variety of tumors, as far as we know, the relationship of KIF18A expression and glioma and its role in the progression and prognosis of glioma have not been well studied and reported. Therefore, in our study, we conducted a comprehensive bioinformatics analysis using multiple data sets to explore the prognostic value of KIF18A in glioma, as well as the related regulatory mechanisms, functions and hypothetical pathways.

In this study, a large number of sample information analysis based on multiple data sets showed that KIF18A expression was increased in a variety of tumor tissues [13,15,35-37] (Figure 1a), and the expression of KIF18A mRNA (Figure 1b) and protein (Figure 7) in glioma was significantly increased. Therefore, KIF18A expression was increased in gliomas, and its relationship with glioma and its function were further verified. By analyzing the information of the three data sets, the results showed that KIF18A could be an independent predictor of the poor prognosis of glioma and significantly reduce the overall survival time for all grades gliomas. Through the analysis of various grades of gliomas, KIF18A overexpression was mainly negatively correlated with OS of grade 3 gliomas. The results are shown in Figure 2 and Figure 3. ROC results showed that KIF18A, as a biomarker of glioma, had significant diagnostic value, as shown in Figure 4. Analysis of the clinical features of gliomas showed that the increase in KIF18A was statistically significant in elderly and high-grade gliomas, compared with lower-age and lower-grade gliomas (Figure 5).. Gene set enrichment analysis showed that KIF18A plays an important role in four signaling pathways, including the KEGG_DNA_REPLICATION , the KEGG_CELL_CYCLE, the KEGG_MISMATCH_REPAIR and the KEGG_NUCLEOTIDE_EXCISION_REPAIR, which have been reported in many tumor researches [38-42], suggesting the potential role of KIF18A as a new therapeutic and prognostic target for glioma.

Although this study has improved our understanding of the relationship between KIF18A and glioma, there are still some limitations. First, multiple public database data make up for the shortcomings of single-center studies, but retrospective studies still have their limitations, especially the unevenness of interventions and the lack of certain information. Therefore, prospective studies should be conducted in future studies to avoid the analysis bias caused by the retrospective nature of current studies. Second, this study shows that the effect of KIF18A overexpression on different grades of gliomas is different, suggesting that KIF18A plays a unique role in subgroup analysis. However, the lack of detailed information of each grade of gliomas is another limitation. It is necessary to further study the long-term predictive effect of KIF18A on different grades of gliomas.

\section{Conclusion}

In conclusion, we found that the expression of KIF18A in glioma was significantly increased, and the high expression of KIF18A was closely related to the progression and poor prognosis of glioma. These data suggest that KIF18A can be used as a target to play a more effective role in the diagnosis and treatment for glioma. Inhibition of KIF18A expression may be a new strategy for the treatment of glioma. 


\section{Abbreviations}

KIF18A: kinesin family member 18A; AUC: The area under ROC curve; ROC: Receiver operating characteristic; OS: Overall Survival; GSEA: Gene set enrichment analysis; KEGG: Kyoto Encyclopedia of Genes and Genomes; CGGA: Chinese Glioma Genome Atlas; TCGA: The Cancer Genome Atlas; GEPIA: Gene Expression Profiling Interactive Analysis

\section{Declarations}

\section{Acknowledgments}

We thank Zhe Jin in the Department of Medical Cell Biology at Uppsala University in

for providing scientific and language editing on this manuscript.

\section{Authors' contributions}

FW and ZL participated in the study design, analysis of data and preparation of the manuscript. HC, YW, YL carried out the data download work and the data collection and interpretation and wrote the first draft. WY, LW, PM, XL, WZ checked the data and discussed statistical analysis. All authors read and approved the final manuscript.

\section{Funding}

This study was partially supported by The Key Research and Development Program of Ningxia (2018BFG02007) and the National Natural Science Foundation of China (NSFC, 81660226)

\section{Availability data and materials}

The data analyzed in this study were obtained from open data including the CGGA, TCGA database and the gene expression omnibus database (GSE4290 and GSE50161).

\section{Ethics approval and consent to participate}

The study was approved by the Ethics Committee of General Hospital of Ningxia Medical University. Data of our present study was obtained from public open database, so there was no informed consent from participants.

\section{Consent for publication}

Not appliable.

\section{Competing interest}

The authors declare that they have no competing interests.

\section{Author details}

${ }^{1}$ Department of Neurosurgery, General Hospital of Ningxia Medical University, Yinchuan, China. ${ }^{2}$ The First Affiliated Hospital of Xinxiang Medical University, Weihui, China. ${ }^{3}$ Department of Orthopaedics, Henan Provincial People's Hospital, People's Hospital of Zhengzhou University, School of Clinical Medicine, Henan University, Zhengzhou, Henan, China

\section{References}

1. Ostrom QT, Gittleman H, Liao P, Vecchione-Koval T, Wolinsky Y, Kruchko C, Barnholtz-Sloan JS. CBTRUS Statistical Report: Primary brain and other central nervous system tumors diagnosed in the United States in 2010-2014. Neuro Oncol. 2017;19:v1-v88. 
2. Haider SA, Asmaro K, Kalkanis SN, Lee IY, Bazydlo M, Nerenz DR, Salloum RG, Snyder J, Walbert T. The Economic Impact of Glioma Survivorship: the cost of care from a patient perspective. Neurology. 2020. 2020; 9 :

10.1212/WNL.0000000000010263.

3. Stupp R, Hegi ME, Mason WP, van den Bent MJ, Taphoorn MJ, Janzer RC, Ludwin SK, Allgeier A, Fisher B, et al. Effects of radiotherapy with concomitant and adjuvant temozolomide versus radiotherapy alone on survival in glioblastoma in a randomised phase III study: 5-year analysis of the EORTC-NCIC trial. Lancet Oncol. 2009;10:459-66.

4. Sanson M, Hosking FJ, Shete S, Zelenika D, Dobbins SE, Ma Y, Enciso-Mora V, Idbaih A, Delattre JY, et al. Chromosome 7p11.2 (EGFR) variation influences glioma risk. Hum Mol Genet. 2011;20:2897-904.

5. Stacey SN, Sulem P, Jonasdottir A, Masson G, Gudmundsson J, Gudbjartsson DF, Magnusson OT, Gudjonsson SA, Sigurgeirsson B, et al. A germline variant in the TP53 polyadenylation signal confers cancer susceptibility. Nat Genet. 2011;43:1098-103.

6. Vale RD, Reese TS, Sheetz MP. Identification of a novel force-generating protein, kinesin, involved in microtubule-based motility. Cell. 1985;42:39-50.

7. Hirokawa N, Noda Y, Tanaka Y, Niwa S. Kinesin superfamily motor proteins and intracellular transport. Nat Rev Mol Cell Biol. 2009;10:682-96.

8. Wordeman L. How kinesin motor proteins drive mitotic spindle function: Lessons from molecular assays. Semin Cell Dev Biol. 2010;21:260-8.

9. Taniuchi K, Nakagawa H, Nakamura T, Eguchi H, Ohigashi H, Ishikawa O, Katagiri T, Nakamura Y. Down-regulation of RAB6KIFL/KIF20A, a kinesin involved with membrane trafficking of discs large homologue 5, can attenuate growth of pancreatic cancer cell. Cancer Res. 2005;65:105-12.

10. Huszar D, Theoclitou ME, Skolnik J, Herbst R. Kinesin motor proteins as targets for cancer therapy. Cancer Metastasis Rev. 2009;28:197-208.

11. Taniwaki M, Takano A, Ishikawa N, Yasui W, Inai K, Nishimura H, Tsuchiya E, Kohno N, Nakamura Y, Daigo Y. Activation of KIF4A as a prognostic biomarker and therapeutic target for lung cancer. Clin Cancer Res. 2007;13:6624-31.

12. Corson TW, Gallie BL. KIF14 mRNA expression is a predictor of grade and outcome in breast cancer. Int $\mathrm{J}$ Cancer. 2006;119:1088-94.

13. Luo W, Liao M, Liao Y, Chen X, Huang C, Fan J, Liao W. The role of kinesin KIF18A in the invasion and metastasis of hepatocellular carcinoma. World J Surg Oncol. 2018;16:36.

14. Kasahara M, Nagahara M, Nakagawa T, Ishikawa T, Sato T, Uetake H, Sugihara K. Clinicopathological relevance of kinesin family member 18A expression in invasive breast cancer. Oncol Lett. 2016;12:1909-1914.

15. Schiewek J, Schumacher U, Lange T, Joosse SA, Wikman H, Pantel K, Mikhaylova M, Kneussel M, Linder S, et al. Clinical relevance of cytoskeleton associated proteins for ovarian cancer. J Cancer Res Clin Oncol. 2018;144:2195-2205.

16. Alfarsi LH, Elansari R, Toss MS, Diez-Rodriguez M, Nolan CC, Ellis IO, Rakha EA, Green AR. Kinesin family member-18A (KIF18A) is a predictive biomarker of poor benefit from endocrine therapy in early ER+ breast cancer. Breast Cancer Res Treat. 2019;173:93-102.

17. Nagahara M, Nishida N, Iwatsuki M, Ishimaru S, Mimori K, Tanaka F, Nakagawa T, Sato T, Sugihara K, et al. Kinesin 18A expression: clinical relevance to colorectal cancer progression. Int J Cancer. 2011;129:2543-52.

18. Menyhárt O, Nagy Á, Győrffy B. Determining consistent prognostic biomarkers of overall survival and vascular invasion in hepatocellular carcinoma. R Soc Open Sci. 2018;5:181006.

19. Wang L, Yang S, Sun R, Lu M, Wu Y, Li Y. Expression of KIF18A in gastric cancer and its association with prognosis. Zhonghua Wei Chang Wai Ke Za Zhi. 2016;19:585-9.

20. Stangeland B, Mughal AA, Grieg Z, Sandberg CJ, Joel M, Nygård S, Meling T, Murrell W, Vik Mo EO, et al. Combined expressional analysis, bioinformatics and targeted proteomics identify new potential therapeutic targets in glioblastoma stem cells. Oncotarget. 2015;6:26192-215. 
21. Marcus Al, Peters U, Thomas SL, Garrett S, Zelnak A, Kapoor TM, Giannakakou P. Mitotic kinesin inhibitors induce mitotic arrest and cell death in Taxol-resistant and -sensitive cancer cells. J Biol Chem. 2005;280:11569-77.

22. Mc Gee MM. Targeting the Mitotic Catastrophe Signaling Pathway in Cancer. Mediators Inflamm. 2015;2015:146282.

23. Myers SM, Collins I. Recent findings and future directions for interpolar mitotic kinesin inhibitors in cancer therapy. Future Med Chem. 2016;8:463-89.

24. Rao CV, Kurkjian CD, Yamada HY. Mitosis-targeting natural products for cancer prevention and therapy. Curr Drug Targets. 2012;13:1820-30.

25. Rath O, Kozielski F. Kinesins and cancer. Nat Rev Cancer. 2012;12:527-39.

26. Liu M, Liu Y, Hou B, Bu D, Shi L, Gu X, Ma Z. Kinesin superfamily protein 17 contributes to the development of bone cancer pain by participating in NR2B transport in the spinal cord of mice. Oncol Rep. 2015;33:1365-71.

27. Wang Q, Wang L, Li D, Deng J, Zhao Z, He S, Zhang Y, Tu Y. Kinesin family member 14 is a candidate prognostic marker for outcome of glioma patients. Cancer Epidemiol. 2013;37:79-84.

28. Thériault BL, Pajovic S, Bernardini MQ, Shaw PA, Gallie BL. Kinesin family member 14: an independent prognostic marker and potential therapeutic target for ovarian cancer. Int J Cancer. 2012;130:1844-54.

29. Sherin L, Farwa S, Sohail A, Li Z, Bég OA. Cancer drug therapy and stochastic modeling of "nano-motors". Int J Nanomedicine. 2018;13:6429-6440.

30. Du Y, English CA, Ohi R. The kinesin-8 Kif18A dampens microtubule plus-end dynamics. Curr Biol. 2010;20:374-80.

31. Luboshits G, Benayahu D. MS-KIF18A, new kinesin; structure and cellular expression. Gene. 2005;351:19-28.

32. Mayr MI, Hümmer S, Bormann J, Grüner T, Adio S, Woehlke G, Mayer TU. The human kinesin Kif18A is a motile microtubule depolymerase essential for chromosome congression. Curr Biol. 2007;17:488-98.

33. Stumpff J, von Dassow G, Wagenbach M, Asbury C, Wordeman L. The kinesin-8 motor Kif18A suppresses kinetochore movements to control mitotic chromosome alignment. Dev Cell. 2008;14:252-62.

34. Diaz-Moralli S, Tarrado-Castellarnau M, Miranda A, Cascante M. Targeting cell cycle regulation in cancer therapy. Pharmacol Ther. 2013;138:255-71.

35. Zhang H, Shen T, Zhang Z, Li Y, Pan Z. Expression of KIF18A Is Associated with Increased Tumor Stage and Cell Proliferation in Prostate Cancer. Med Sci Monit. 2019;25:6418-6428.

36. Li X, Liu M, Zhang Z, Zhang L, Liang X, Sun L, Zhong D. High kinesin family member 18A expression correlates with poor prognosis in primary lung adenocarcinoma. Thorac Cancer. 2019;10:1103-1110.

37. Chen FT, Zhong FK. Kinesin Family Member 18A (KIF18A) Contributes to the Proliferation, Migration, and Invasion of Lung Adenocarcinoma Cells In Vitro and In Vivo. Dis Markers. 2019;2019:6383685.

38. Ubhi T, Brown GW. Exploiting DNA Replication Stress for Cancer Treatment. Cancer Res. 2019;79:1730-1739.

39. Ingham M, Schwartz GK. Cell-Cycle Therapeutics Come of Age. J Clin Oncol. 2017;35:2949-2959.

40. Baretti M, Le DT. DNA mismatch repair in cancer. Pharmacol Ther. 2018;189:45-62.

41. Marteijn JA, Lans $\mathrm{H}$, Vermeulen W, Hoeijmakers $\mathrm{JH}$. Understanding nucleotide excision repair and its roles in cancer and ageing. Nat Rev Mol Cell Biol. 2014;15:465-81.

42. Jager M, Blokzijl F, Kuijk E, Bertl J, Vougioukalaki M, Janssen R, Besselink N, Boymans S, Ligt J, et al. Deficiency of nucleotide excision repair is associated with mutational signature observed in cancer. Genome Res. 2019;29:1067-1077.

\section{Figures}


a

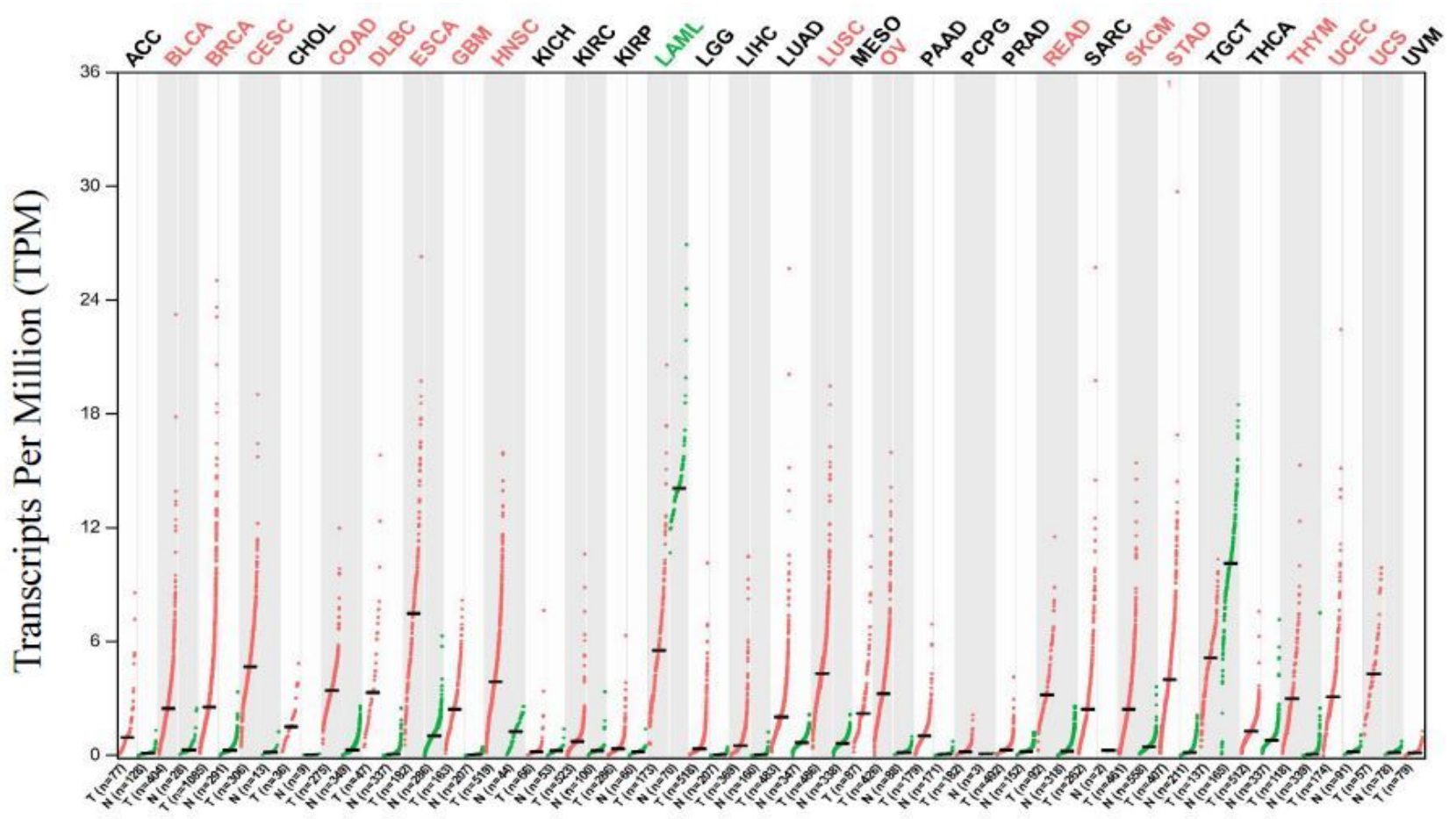

b

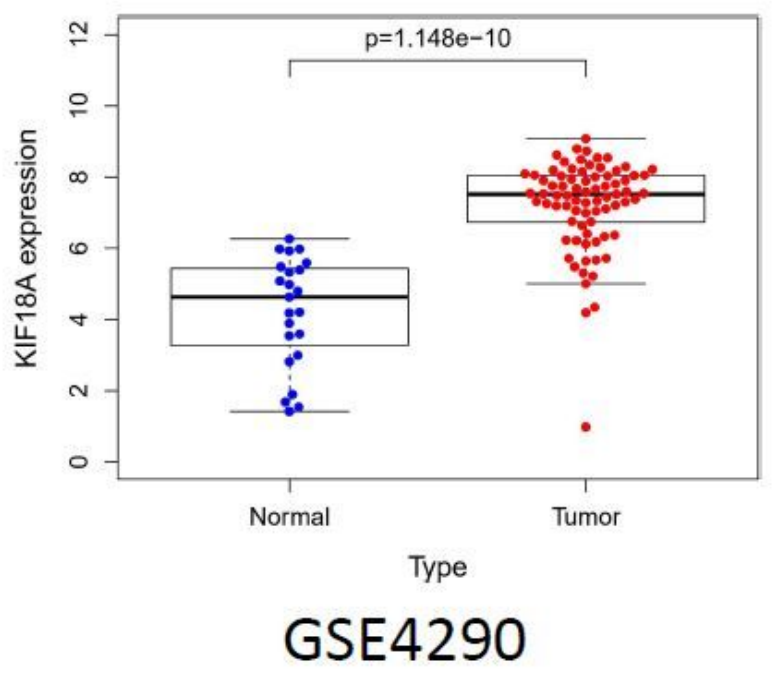

c

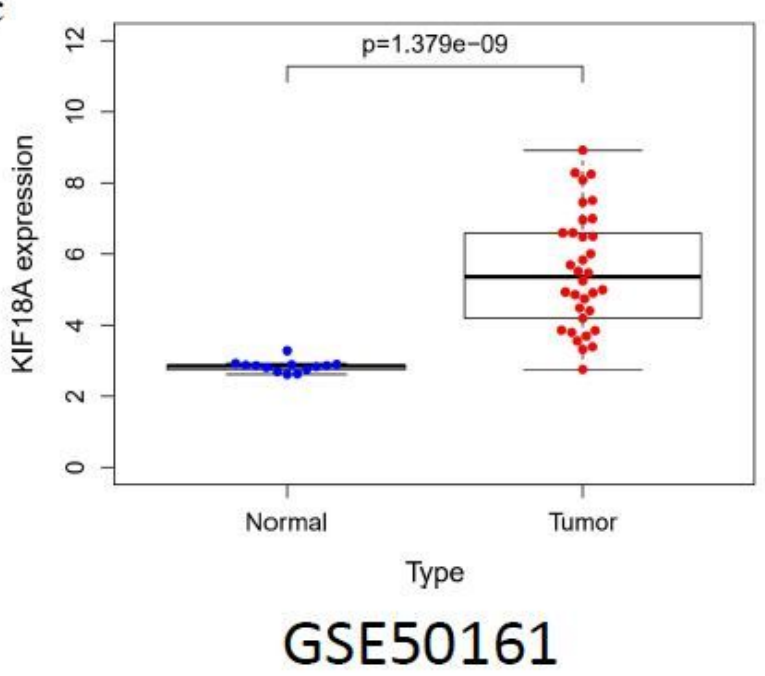

Figure 1

Page 10/15 


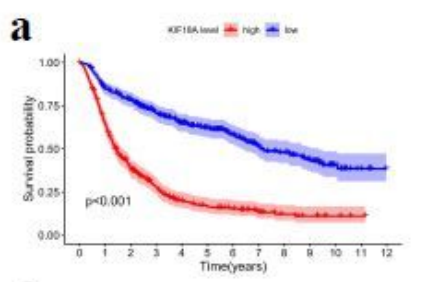

kEp

CGGA RNA-seq

e

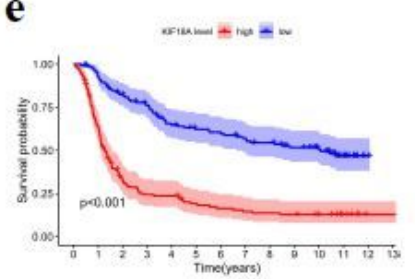

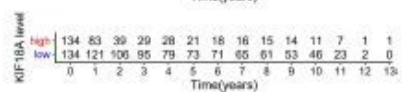

CGGA microarray

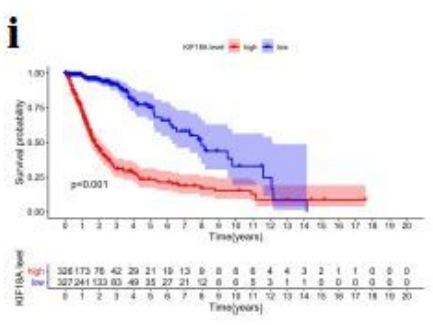

TCGA RNA-seq

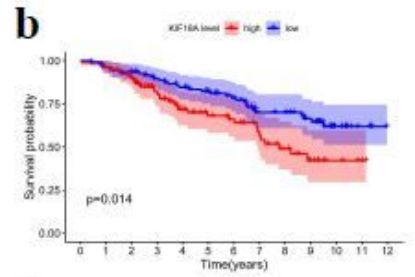

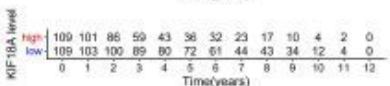

CGGA RNA-seq

(Grade II)

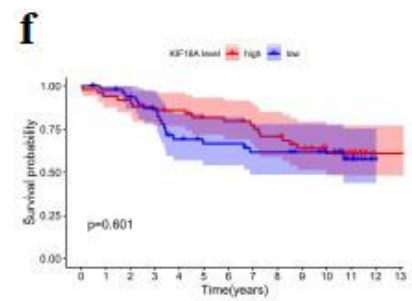

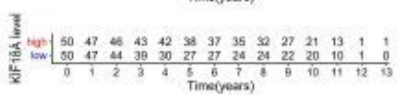

CGGA microarray

(Grade II)

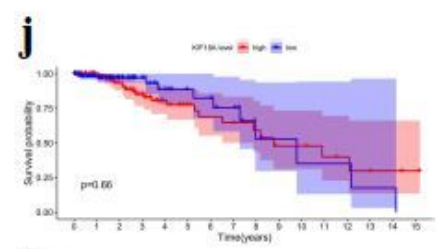

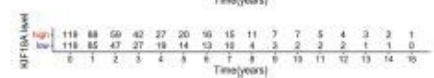

TCGA RNA-seq

(Grade II)

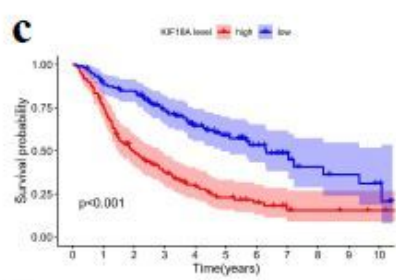

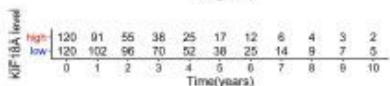

CGGA RNA-seq

(Grade III)

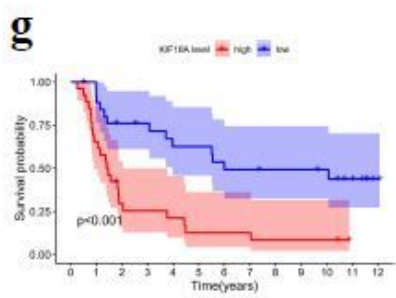

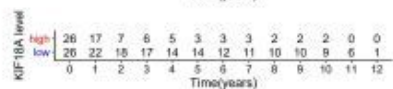

CGGA microarray

(Grade III)

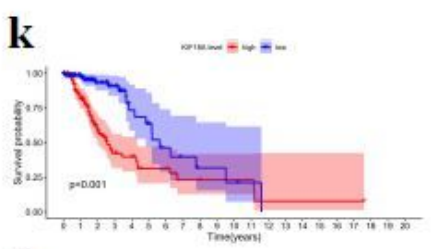

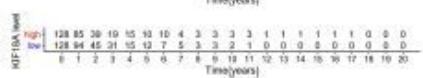

TCGA RNA-seq

(Grade III)

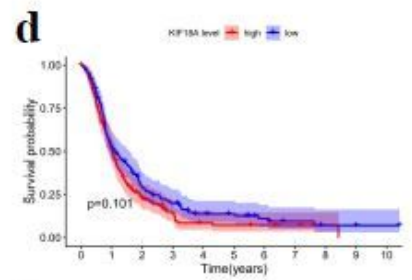

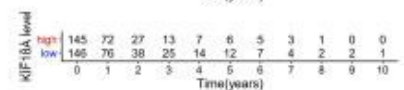

CGGA RNA-seq

(Grade IV)

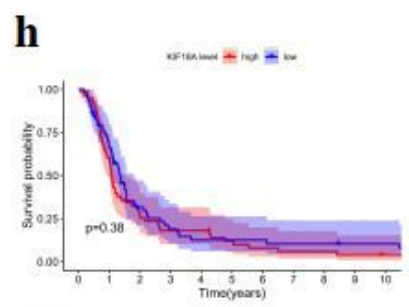

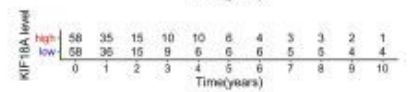

CGGA microarray

(Grade IV)
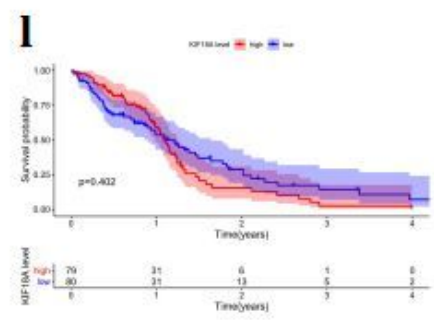

TCGA RNA-seq

(Grade IV)

Figure 2 
$\mathbf{a}$

\begin{tabular}{|c|c|c|}
\hline & pvalue & Hazard ratio \\
\hline KIF18A & $\infty .001$ & $2.201(1.954-2.479)$ \\
\hline PRS_type & $\infty .001$ & $2.123(1.818-2.478)$ \\
\hline Histology & $\$ .001$ & $4.487(3.696-5.449)$ \\
\hline Grade & $\infty .001$ & $2883(2.528-3.291)$ \\
\hline Gender & 0.655 & $1.044(0.856-1.258)$ \\
\hline Age & $\infty .001$ & $1.624(1.345-1.960)$ \\
\hline Radio & 0.571 & $0.929(0.720-1.199)$ \\
\hline Chemo & $\infty .001$ & $1.647(1.328-2.044)$ \\
\hline IDH_mutation & $\$ .001$ & $0.317(0.262-0.384)$ \\
\hline $1 \mathrm{p} 19 \mathrm{q}$ codeletion & $\infty 001$ & $0231(0.169-0.315)$ \\
\hline
\end{tabular}

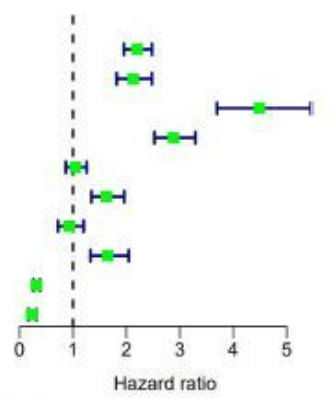

\section{CGGA RNA-seq (univariate)}

c

\begin{tabular}{|c|c|c|}
\hline & pvalue & Hazard ratio \\
\hline KIF18A & $\infty .001$ & $1.649(1.486-1.830)$ \\
\hline TCGA_sublypes & $\infty .001$ & $0.632(0.539-0.742)$ \\
\hline PRS_type & $\$ 0.001$ & $2042(1.511-2.759)$ \\
\hline Histoiogy & $\infty .001$ & $4.437(3.235-6.006)$ \\
\hline Grade & $\infty .001$ & $2.567(2.125-3.100)$ \\
\hline Gender & 0.125 & $1.289(0.936-1.720)$ \\
\hline Age & $\infty 0.001$ & $1.736(1.293-2.349)$ \\
\hline Radio & 0.003 & $0.495(0.313-0.782)$ \\
\hline Chems & 0.007 & $1.530(1.125-2.079)$ \\
\hline & 0.00 & $3309-0$ \\
\hline
\end{tabular}

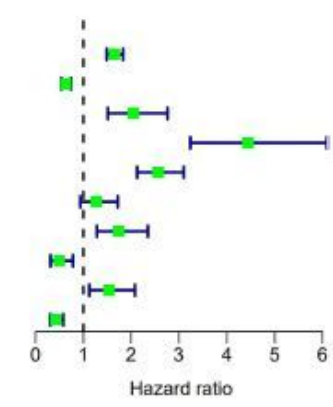

CGGA microarray (univariate)

e
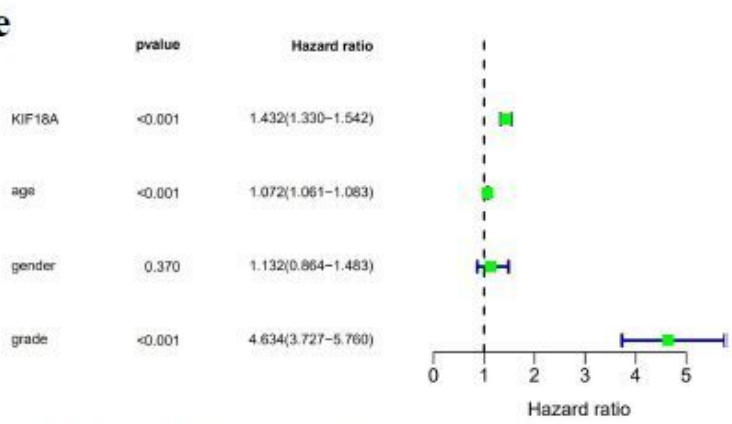

TCGA RNA-seq (univariate) b
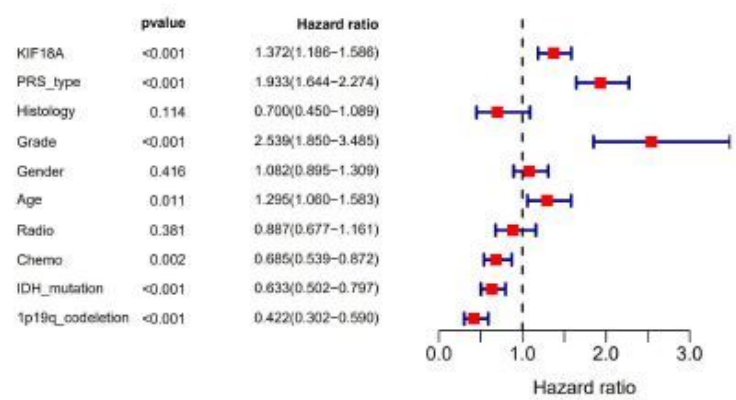

CGGA RNA-seq (mutivariate)

d $\begin{array}{lrr} & \text { pvalue } & \text { Hazard ratio } \\ \text { KIF182 } & \infty .001 & 1.372(1.203-1.566) \\ \text { TCGA_subtypes } & 0.161 & 0.889(0.754-1.048) \\ \text { PRS_type } & 0.001 & 1.725(1.233-2.413) \\ \text { Histology } & 0.058 & 0.451(0.198-1.027) \\ \text { Grade } & \infty .001 & 2.641(1.537-4.537) \\ \text { Gender } & 0.903 & 0.981(0.715-1.345) \\ \text { Mge } & 0.470 & 1.130(0.812-1.572) \\ \text { Radio } & 0.003 & 0.464(0.279-0.774) \\ \text { Chemb } & 0.588 & 0.909(0.644-1.283) \\ \text { IDH_mutation } & 0.612 & 0.902(0.605-1.344)\end{array}$

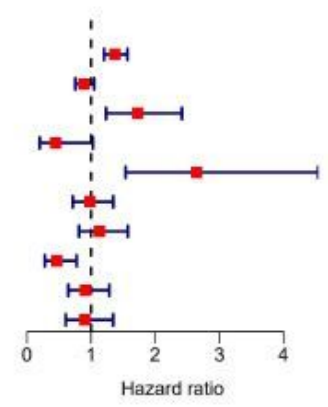

CGGA microarray (mutivariate)

f

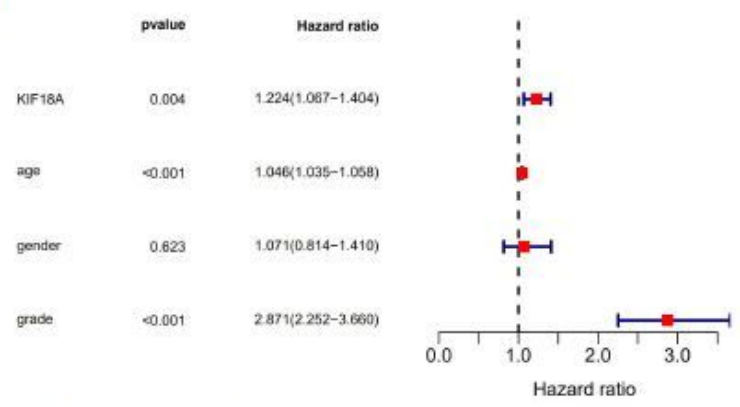

TCGA RNA-seq (mutivariate)

Figure 3 
a

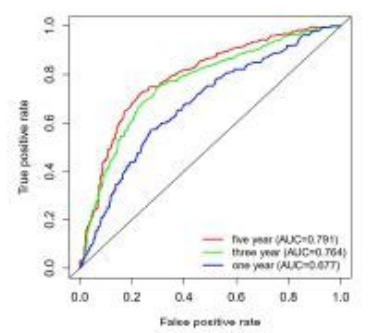

CGGA RNA-seq

e

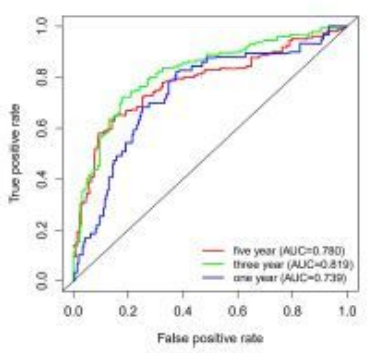

CGGA microarray

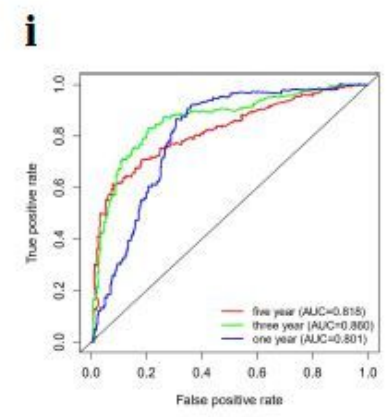

TCGA RNA-seq b

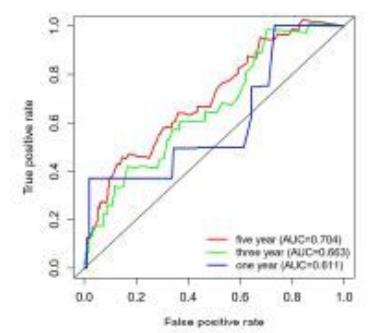

CGGA RNA-seq

(Grade II)

f

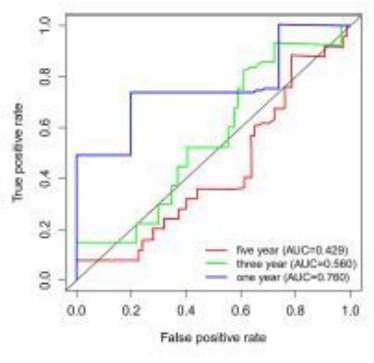

CGGA microarray

(Grade II)

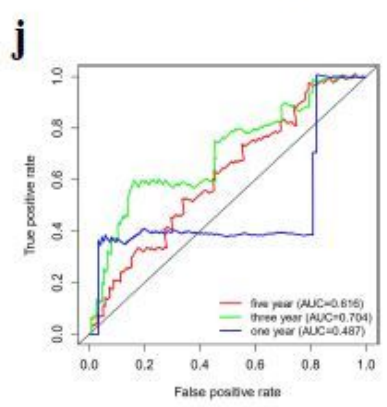

TCGA RNA-seq

(Grade II) c

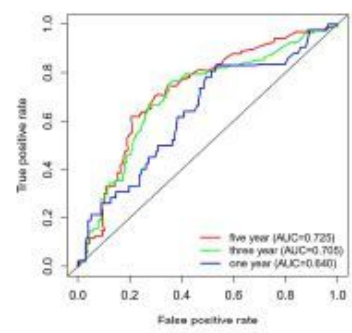

CGGA RNA-seq

(Grade III)

g

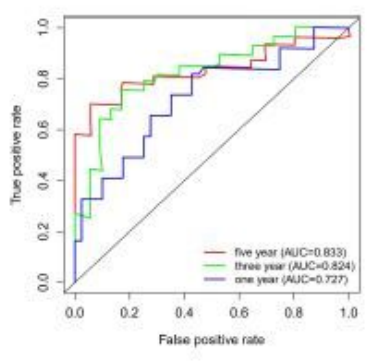

CGGA microarray

(Grade III)

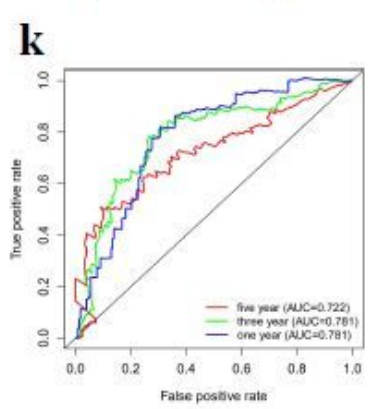

TCGA RNA-seq (Grade III) d

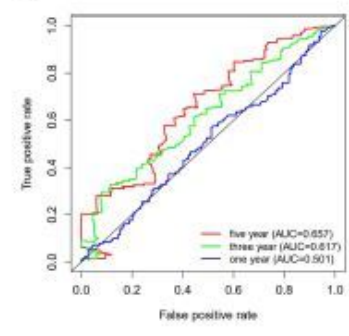

CGGA RNA-seq

h (Grade IV)

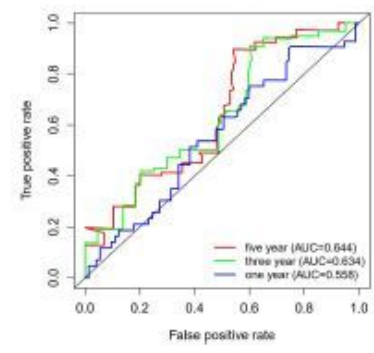

CGGA microarray (Grade IV)

I

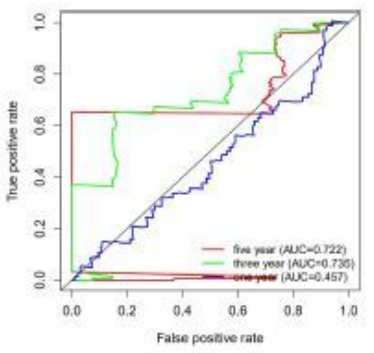

TCGA RNA-seq (Grade IV)

Figure 4 

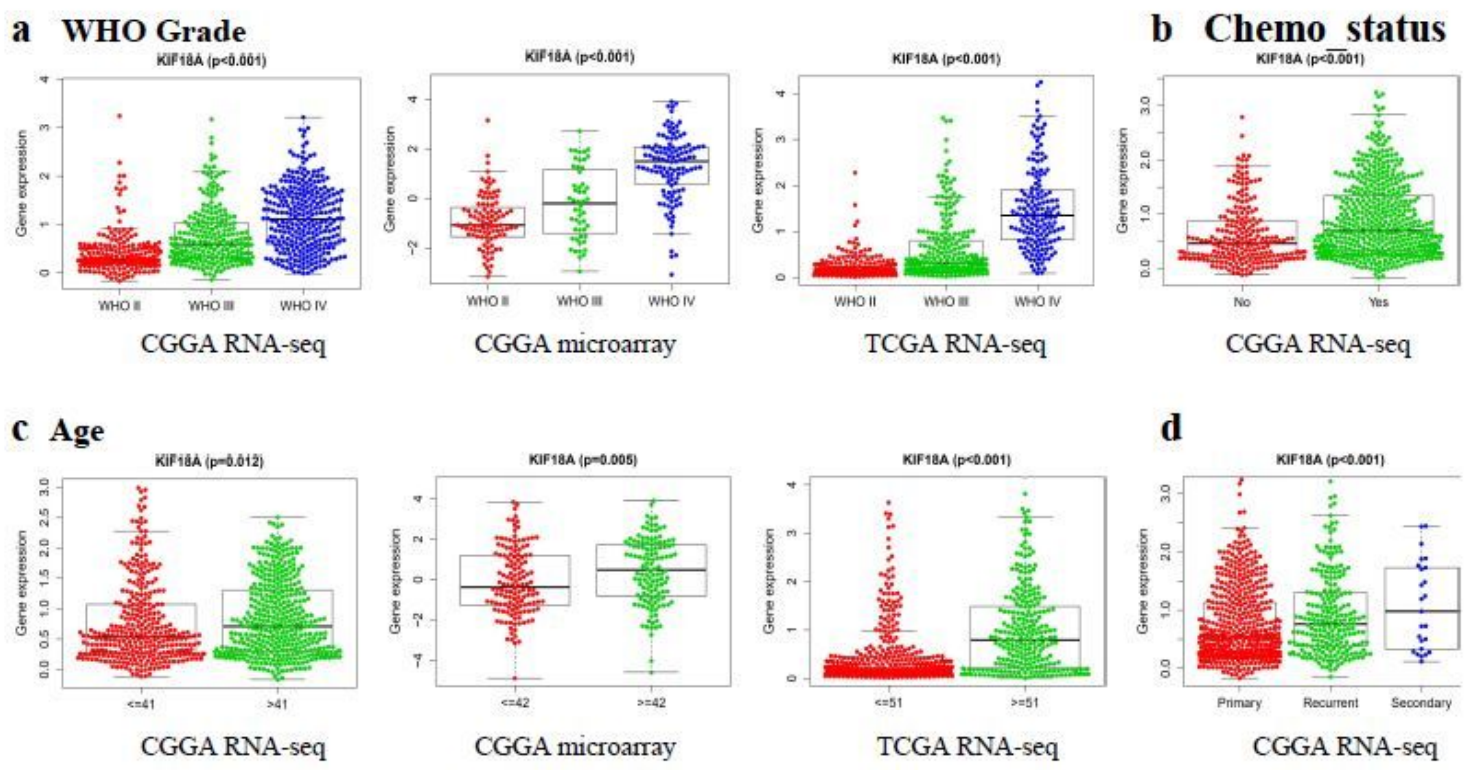

e IDH Mutation

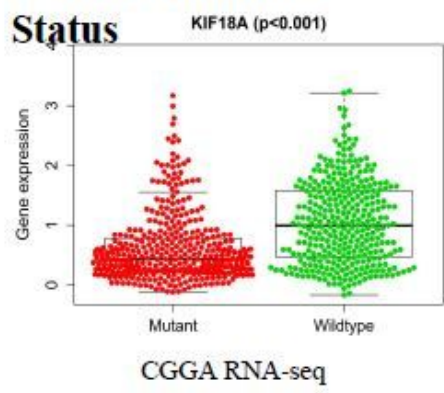

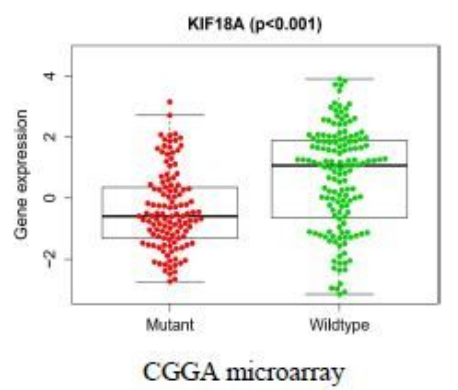

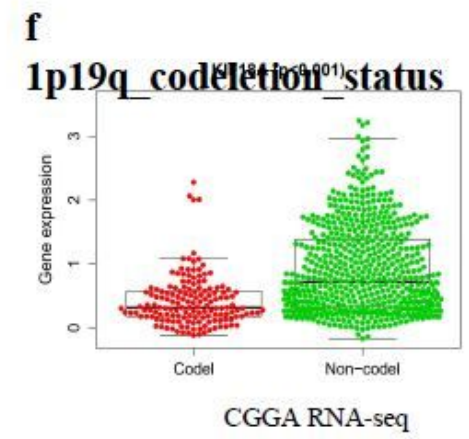

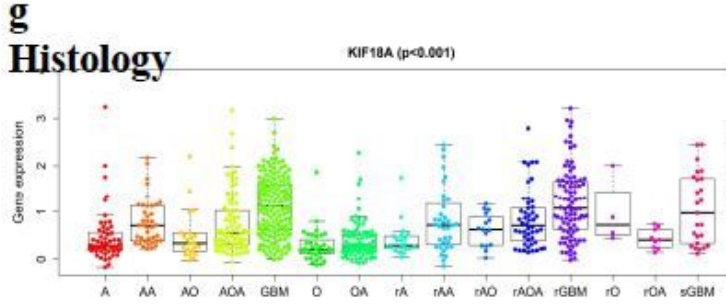

CGGA RNA-seq

\section{d}

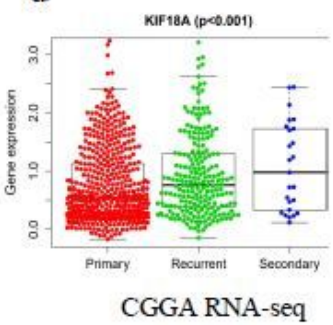



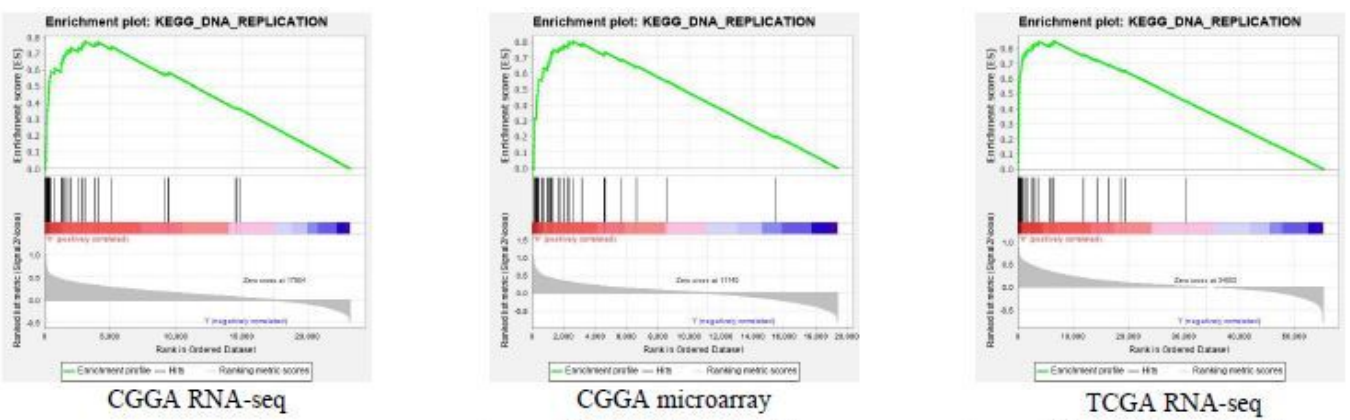

b
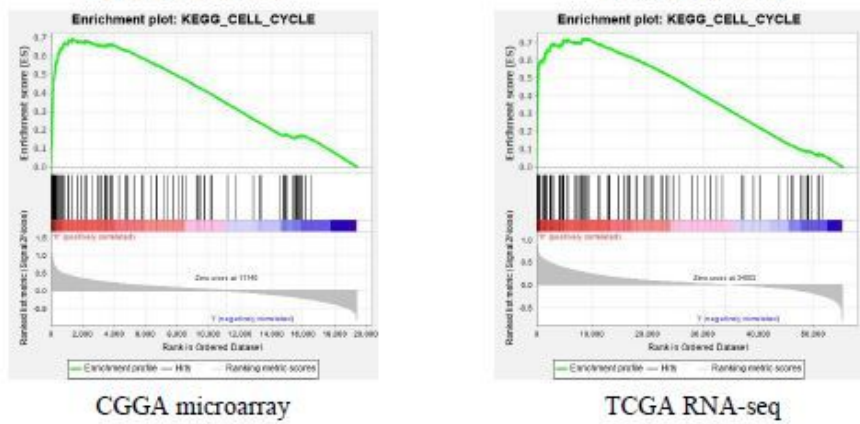

C

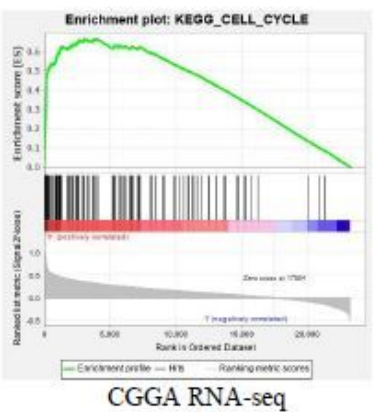

CGGA microarray

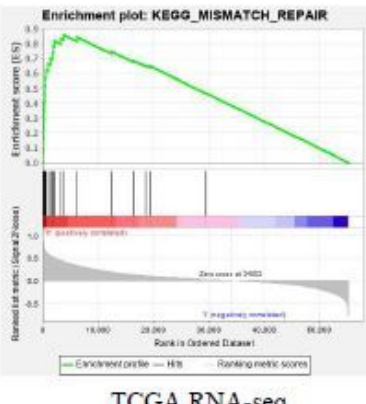

TCGA RNA-seq
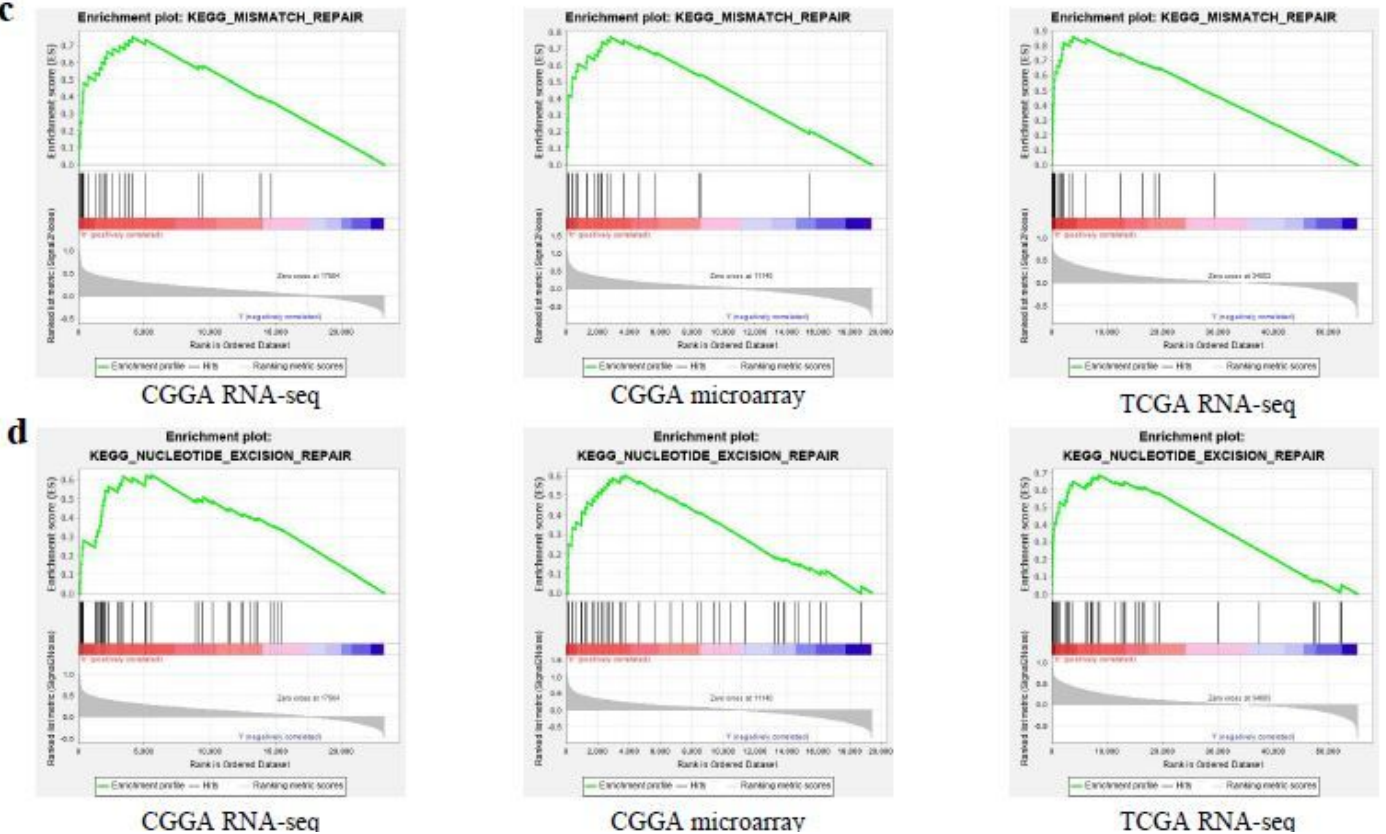

Figure 7

\section{Supplementary Files}

This is a list of supplementary files associated with this preprint. Click to download.

- TableS3TCGA.docx

- Tables2CGGAseq.docx

- TableS1CGGAmicroarray.docx 